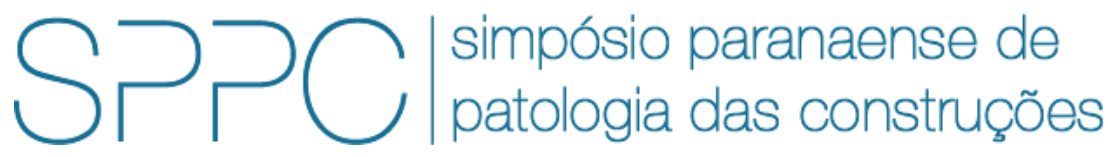

ISSN 2526-7248 artigo 2SPPC1016, pp. 185-198, 2017

\title{
Avaliação da influência do empacotamento dos agregados na durabilidade de concretos através de ensaios não destrutivos: resistividade elétrica e propagação de ondas de ultrassom
}

\author{
LENZ, L. A. ${ }^{1}$, LONDERO, C. ${ }^{1}$, KLEIN, N. S. ${ }^{2}$ e MAZER, $W^{3}$. \\ ${ }^{1}$ Mestre, Universidade Federal do Paraná, lenzlauri@hotmail.com; carolinalondero7@gmail.com \\ 2Doutora, Universidade Federal do Paraná, nayaraklein@ufpr.br \\ 32Doutor, Universidade Tecnológica Federal do Paraná, mazer.eng@gmail.com
}

\begin{abstract}
Resumo: Observa-se nos últimos anos uma grande preocupação do meio técnico de engenharia no sentido de incentivar projetos ligados à durabilidade e ao prolongamento da vida útil das estruturas de concreto armado em função dos problemas de degradação precoce que as estruturas vêm sofrendo. Os ensaios não destrutivos tornam-se uma alternativa para verificar a presença de possíveis descontinuidades no concreto, sem alterar as suas características físicas, mecânicas ou químicas, e sem interfirir na sua utilização posterior. Dentre esses ensaios, destacam-se os ensaios de resistividade elétrica e de módulo de elasticidade dinâmico através de ultrassom. O presente trabalho tem por objetivo avaliar a influência do empacotamento dos agregados, areia e brita, na resistividade elétrica e propagação de ondas ultrassônicas de concretos convencionais, com resistências de 25 e $40 \mathrm{MPa}$. Para isso, foram produzidos concretos com a estrutura granular modificada utilizando técnicas de empacotamento de partículas. Estudou-se, também, a influência do teor de agregados na resistividade elétrica e no módulo de elasticidade dinâmico, por meio do aumento do teor de agregados em relação ao cimento. Como resultado, observou-se que, ao modificar a estrutura granular dos concretos, mantendo a mesma resistência mecânica, os resultados de resistividade elétrica se mantiveramestatisticamente equivalentes. Já os resultados de módulo de elasticidade dinâmico aumentaram conforme a densidade de empacotamento aumentou. Ao elevar o teor de agregados em relação ao cimento percebe-se que houve aumento dos valores tanto de resistividade elétrica quanto do módulo de elasticidade dinâmico. Ressalta-se também que tanto o empacotamento da estrutura granular quanto o aumento do teor de agregados em relação ao cimento contribuiu para a densificação do concreto.
\end{abstract}

Palavras-chave: Empacotamento de partículas. Resistividade elétrica. Módulo de elasticidade dinâmico. Ultrassom. Agregados.

\begin{abstract}
In the last few years, there is a great concern of the engineering technician in order to encourage projects related to the durability and the prolongation of the service live of the reinforced concrete structures due to the problems of early degradation that the structures have been suffering. The non-destructive tests become an alternative to verify the presence of possible discontinuities in concrete, without altering their physical, mechanical or chemical characteristics, and without interfering in their later use. Among these tests, the electrical resistivity and ultrasound wave propagation velocity tests are outstanding. The objective of the present work is toevaluatethe influence of the packing of the aggregates, sand and gravel, on the electrical resistivity and propagation of ultrasonic waves of conventional concretes, with compressive strengths of 25 and $40 \mathrm{MPa}$. For this, concretes with modified granular structure were produced using particle packaging techniques. It was also studied the influence of the aggregate content on the electrical resistivity and dynamic modulus, by increasing the aggregate content in relation to the cement. As a result, it was observed that by modifying the granular structure of the concrete, maintaining the same mechanical strength, the results of electrical resistivity werestatistically equivalent. On the other hand,the results of dynamic modulushave increased with increasing packing density. When increasing the content of aggregates in relation to cement it wasnoticed that there was an increase in the values of both electrical resistivity and dynamic modulus. It is also highlighted that both the packing of the granular structure and the increase of the aggregate content contributed to the densification of the concrete.
\end{abstract}

Keywords: Particle packing. Electrical resistivity. Dynamic modulus of elasticity. Ultrasound wave propagation velocity tests. Aggregates. 


\section{Introdução}

Está cada vez mais crescente entre os profissionais da área da construção civil o estudo para garantir maior durabilidade das estruturas de concreto, devido aos custos elevados de reparo e manutenção de estruturas existentes. Neville e Brooks [1] estimam que, nos países desenvolvidos, esses custos alcancem a margem de $40 \%$ de todos os investimentos da indústria da construção civil. Assim, a busca pelo incremento da durabilidade passa a ser um quesito essencial no planejamento de estruturas a longo prazo.

Segundo a ABNT NBR 6118 [2], a durabilidade do concreto pode ser definida como a capacidade de uma determinada estrutura resistir às influências ambientais previstas e definidas na fase de projeto.

O ACI Commitee 201 também atribui à durabilidade do concreto de cimento Portland a propensão de resistir à ação de intempéries, ataques químicos, abrasão ou qualquer outro processo de deterioração. Por conseguinte, um concreto durável irá preservar sua forma, qualidade e utilização original quando exposto ao ambiente para o qual foi projetado, sendo necessário também estabelecer métodos para monitorar 0 comportamento das estruturas [3].

Assim, a verificação de parâmetros de durabilidade consiste em uma forma de avaliar o concreto, principalmente quando há alterações significativas na sua composição, como, por exemplo, a redução de cimento Portland. Nesse âmbito podem-se citar como relevantes a verificação dos fenômenos de carbonatação, de ação de íons cloreto e a realização de ensaios não destrutivos que se correlacionem com a durabilidade do concreto.

Os ensaios não destrutivos são técnicas que podem ser utilizadas na inspeção de estruturas de concreto armado para avaliar a qualidade de um concreto em relação à durabilidade. Estes permitem verificar a existência de possíveis problemas, sem causar danos ao elemento ensaiado. Existem várias técnicas de ensaio como a mensuração da resistividade elétrica e da velocidade de propagação de ondas de ultrassom.

O interesse pelo empacotamento de partículas aumentou nas diversas áreas de engenharia, uma vez que grande parte dos materiais industriais e naturais utilizados pelo homem é composto por partículas de diferentes formas e tamanhos [4].

O presente trabalho tem como objetivo avaliar a influência do empacotamento de partículas dos agregados, areia e brita, na resistividade elétrica e na velocidade de propagação de ondas de ultrassom de concretoscom resistências de 25 e $40 \mathrm{MPa}$. Além disso, buscou-se avaliar ainda a variação do teor de agregados $(\mathrm{m})$ na mensuração da resistividade elétrica e da velocidade de propagação de ondas de ultrassom, buscando avaliar a qualidade dos concretos em função do aumento do teor de agregados. 


\section{Empacotamento de Partículas}

Uma mistura de concreto pode ser entendida como um acondicionamento seco de partículas de agregados, cujos espaços vazios são preenchidos com pasta de cimento. Dado que essa matriz é mais cara e, normalmente, menos forte e durável do que uma rocha natural, a estratégia básica de dosagem é projetar uma mistura granular prevendo uma porosidade mínima dos agregados. Paralelamente, a composição da matriz pode ser fixada levando em conta a resistência e a durabilidade, sendo a relação água/cimento o parâmetro chave desse processo [5].

A dosagem de um concreto deve visar uma distribuição otimizada das partículas que compõe o material, proporcionando uma mistura com o mínimo de vazios e com alta densidade. Para aumentar a densidade diminuem-se os espaços entre as partículas, preenchendo-os com partículas menores, procurando, assim, estabelecer um empacotamento [6].

Através do empacotamento de partículas é possível melhorar o esqueleto granular que compõem os concretos, considerando tanto os agregados como os materiais finos componentes. Esta otimização do esqueleto granular permite reduzir o consumo de cimento Portland, minimizando a porosidade e, consequentemente, aumentando a resistência mecânica e a durabilidade dos concretos [7].

Ao se tratar dessa otimização, um conceito particularmente importante é o da densidade de empacotamento de um conjunto granular, a qual pode ser definida como o volume de sólidos presentes em uma unidade de volume total. Ela depende de fatores como a forma dos grãos, a distribuição granulométrica das partículas e também dos métodos de compactação aplicados. Logo, para otimizar a densidade de empacotamento, as partículas devem ser selecionadas a fim de minimizar o volume de vazios para obter uma estrutura granular densa e rígida. É importante ressaltar que os modelos de empacotamento utilizam relações e proporções volumétricas de partículas e ocupação de espaço [8-9].

\section{Ensaios Não Destrutivos}

Ensaios não destrutivos são ensaios efetuados na inspeção de materiais e equipamentos, com o objetivo de verificar a presença de possíveis descontinuidades, sem alterar as suas características físicas, mecânicas ou químicas, e sem interfirir na sua utilização posterior [10]. Dentre esses ensaios, destacam-se os ensaios de resistividade elétrica e de velocidade de propagação de ondas de ultrassom.

\subsection{Resistividade Elétrica}

A resistividade elétrica consiste em uma propriedade que caracteriza a dificuldade com que os íons se movimentam no concreto, controlando sua difusão pelo concreto através da solução aquosa presente nos poros, sendo sensível ao teor de umidade de equilíbrio e à temperatura [11-12]. Essa propriedade, quando em conjunto com o processo de oxigenação das barras de aço da armadura, tende a controlar o processo eletroquímico de corrosão. Assim, a velocidade de corrosão das armaduras, e por consequência, a durabilidade das estruturas, estão diretamente ligadas à resistividade elétrica. As barras de aço imersas no concreto são mais propensas a 
LENZ, L. A.;ET AL.. AVALAÇÃO DA INFLUÊNCIA DO EMPACOTAMENTO DOS AGREGADOS NA DURABILIDADE DE CONCRETOS ATRAVÉS DE ENSAIOS NÃO DESTRUTIVOS: RESISTIVIDADE ELÉTRICA E PROPAGAÇÃO DE ONDAS DE ULTRASSOM. $2^{\circ}$ Simpósio Paranaense de Patologia das Construções (2० SPPC), artigo 2SPPC1016, pp. 185-198, 2017. DOI: $10.4322 / 2$ SPPC. 2017.016

atingir um estado de corrosão quando a resistividade é inferior a $10 \mathrm{k} \Omega . \mathrm{cm}$, e tendem a manter um comportamento passivo quando a resistividade do concreto é superior a $30 \mathrm{k} \Omega . \mathrm{cm}[13]$.

Cascudo [13], assim como Whitin e Nagi [14], estabelece que uma resistividade abaixo de $5 \mathrm{k} \Omega$.cm indica uma probabilidade de corrosão muito alta, entre 5 a 10 $\mathrm{k} \Omega$.cm alta, entre 10 a $20 \mathrm{k} \Omega . \mathrm{cm}$ baixa probabilidade e acima de $20 \mathrm{k} \Omega . \mathrm{cm}$ o risco de corrosão torna-se desprezível.

Hoppe [15] destaca que a relação água/aglomerante é o principal parâmetro controlador das características do concreto que atua alterando a estrutura porosa da matriz hidratada. Dessa forma, a resistividade elétrica tende a reduzir com o aumento da porosidade provocada pela elevação da relação de água/aglomerante.

A verificação da resistividade elétrica do concreto pode se dar por diversos métodos, sendo o método dos quatro eletrodos, denominado de método de Wenner, um dos mais utilizados [16]. Uma vantagem dessa técnica é que a resistividade medida refere-se à região do concreto localizada entre os dois eletrodos internos. A influência do agregado, neste caso, pode ser minimizada a partir do ponto que o espaçamento entre os eletrodos internos excede o tamanho máximo do agregado [17]. Um espaçamento de $50 \mathrm{~mm}$ é suficiente para a obtenção de medidas de resistividade elétrica relativamente precisas em grande parte das estruturas de concreto [18].

ensaio consiste em aplicar uma corrente alternada, entre dois eletrodos externos, mensurando a diferença de potencial entre os dois eletrodos internos. Assim, a resistividade elétrica do concreto pode ser calculada pela Eq. 01.

$$
\rho=2 \cdot \pi \cdot \alpha \cdot \frac{U}{I}
$$

Em que: $\rho$ é a resistividade do concreto ( $\Omega . c m)$; $U$ é a tensão medida (Volts); I é a corrente aplicada (Ampere); a é a distância entre eletrodos $(\mathrm{cm})$.

\subsection{Módulo de Elasticidade Dinâmico através de Ultrassom}

Uma das técnicas não destrutivas que vem sendo utilizadas para a avaliação da qualidade de estruturas de concreto é a aplicação de ondas ultrassônicas. $O$ ensaio consiste em aplicar um pulso ultrassônico a fim de mensurar o tempo que esse pulso leva para atravessar a seção de concreto. Ao dividir o comprimento existente entre os transdutores pelo tempo de propagação do pulso obtêm-se a velocidade média da propagação da onda, sendo esse resultado influenciado pelas propriedades do material [19].

O ultrassom tem sua utilização relacionada ao fato de ser possível estabelecer o módulo de elasticidade dinâmico do concreto de forma não destrutiva. Além disso, esse ensaio possibilita estimar a uniformidade e presença de vazios detectados devido às diferenças de velocidade de propagação das ondas, as quais normalmente variam na frequência de $20 \mathrm{kHz}$ a $150 \mathrm{kHz}$. Para concretos convencionais a velocidade de pulso geralmente fica entre 3700 a $4200 \mathrm{~m} / \mathrm{s}$, com uma distância de transdutores que pode variar de 10 a $30 \mathrm{~cm}$ [20]. 
LENZ, L. A.;ET AL.. AVALAÇÃO DA INFLUÊNCIA DO EMPACOTAMENTO DOS AGREGADOS NA DURABILIDADE DE CONCRETOS ATRAVÉS DE ENSAIOS NÃO DESTRUTIVOS: RESISTIVIDADE ELÉTRICA E PROPAGAÇÃO DE ONDAS DE ULTRASSOM. $2^{\circ}$ Simpósio Paranaense de Patologia das Construções (2० SPPC), artigo 2SPPC1016, pp. 185-198, 2017. DOI: 10.4322/2SPPC.2017.016

No Brasil, as recomendações para execução do ensaio com pulso ultrassônico estão prescritas na NBR 8802 [21], a qual descreve sucintamente o ensaio, estabelecendo condições de preparação da superfície do concreto e tipos de transmissão das ondas. Existem três tipos de transmissão para a realização do ensaio de ultrassom, sendo elas direta, indireta e semidireta. Estas dependem das dimensões da estrutura, da precisão requerida e da variabilidade do concreto.

Segundo a NBR 8802 [21], a transmissão direta consiste na configuração mais recomendada, pois permite às ondas serem recebidas com maior intensidade. Os outros tipos de transmissão só devem ser realizados quando não for possível aplicar a transmissão direta.

A norma americana ASTM C 597 [22] demonstra pela Eq. 02 o cálculo da velocidade da onda considerando as propriedades do concreto.

$$
V=\sqrt{\frac{E_{d .}(1-v)}{\rho_{\text {conc }} \cdot(1+v) \cdot(1-2 v)}}
$$

Em que: $V$ é a velocidade da onda $(\mathrm{km} / \mathrm{s})$; $E_{d}$ é o módulo de elasticidade dinâmico $\left(\mathrm{kN} / \mathrm{mm}^{2}\right)$; $\rho_{\text {conc }}$ é a massa específica do concreto $\left(\mathrm{kg} / \mathrm{m}^{3}\right)$; v é o coeficiente de Poisson dinâmico (-).

Para determinar o módulo de elasticidade dinâmico a partir de leituras com ultrassom é necessário, então, conhecer a massa específica do concreto e o coeficiente de Poisson dinâmico. O primeiro pode ser determinado experimentalmente, no concreto em seu estado endurecido, conforme recomendações da NBR 9833 [23], e os resultados normalmente variam de $2.000 \mathrm{~kg} / \mathrm{m}^{3}$ a $2.800 \mathrm{~kg} / \mathrm{m}^{3}$. Para tensões de compressão menores que $0,5 \mathrm{f}_{\mathrm{c}}$ (resistência à compressão do concreto na idade de 28 dias) e tensões de tração menores que $\mathrm{f}_{\mathrm{ct}}$ (resistência à tração do concreto na idade de 28 dias), o coeficiente de Poisson, que varia de 0,11 a 0,21 , pode ser tomado como igual a $0,2[02]$.

\section{Programa Experimental}

Foram consideradas três variáveis para elaboração do programa experimental:

- Resistência à compressão do concreto convencional (classe C25 e classe C40);

- Estrutura granular (referência e modificada pelo empacotamento de partículas);

- Relação agregados secos/cimento (5,08; 6,08 e 7,08 para C25 e 3,08; 4,08 e 5,08 para C40)

As classes de resistência do concreto (fck) de 25 e $40 \mathrm{MPa}$ foram escolhidos em função de abrangerem as principais resistências utilizadas para concretos estruturais convencionais. Buscou-se variar o valor da relação agregado seco/cimento $(\mathrm{m}) \mathrm{a}$ partir do valor encontrado para os traços com a estrutura granular modificada, para 
LENZ, L. A.;ET AL.. AVALAÇÃO DA INFLUÊNCIA DO EMPACOTAMENTO DOS AGREGADOS NA DURABILIDADE DE CONCRETOS ATRAVÉS DE ENSAIOS NÃO DESTRUTIVOS: RESISTIVIDADE ELÉTRICA E PROPAGAÇÃO DE ONDAS DE ULTRASSOM. $2^{\circ}$ Simpósio Paranaense de Patologia das Construções (2० SPPC), artigo 2SPPC1016, pp. 185-198, 2017. DOI:

ambos os concretos de classe C25 e C40, tendo sido usados os valores $(m+1)$ e $(m$ $+2)$.

O cimento Portland utilizado na pesquisa é o CP II-F-32, comumente usado na produção de concreto convencional dosado em central. A caracterização química e físico-mecânica deste material é dada pelo fabricante, conforme apresentado na Tabela 01.

Tabela 01: Características físicas, químicas e mecânicas do CPII-F 32

\begin{tabular}{|c|c|c|c|c|c|c|c|c|c|c|c|}
\hline \multicolumn{12}{|c|}{ Características químicas (\%) } \\
\hline $\mathrm{CaO}$ & $\mathrm{SiO}_{2}$ & $\mathrm{Al}_{2} \mathrm{O}_{3}$ & $\mathrm{Fe}_{2} \mathrm{O}_{3}$ & \multicolumn{2}{|c|}{$\mathrm{MgO}$} & \multicolumn{2}{|c|}{$\mathrm{SO}_{3}$} & $\begin{array}{l}\mathrm{CaO} \\
\text { livre }\end{array}$ & $\begin{array}{l}\text { Perda } \\
\text { ao fogo }\end{array}$ & \multicolumn{2}{|c|}{$\begin{array}{l}\text { Resíduo } \\
\text { Insolúve }\end{array}$} \\
\hline 60,30 & 18,73 & 4,32 & 2,76 & & 87 & & 60 & 1,36 & 4,65 & & 1,32 \\
\hline \multicolumn{12}{|c|}{ Características físicas } \\
\hline \multirow{2}{*}{$\begin{array}{c}\text { Massa } \\
\text { esp. }(\mathrm{g} / \\
\left.\mathrm{cm}^{3}\right)\end{array}$} & \multirow{2}{*}{$\begin{array}{l}\text { Início } \\
\text { pega } \\
\text { (h) }\end{array}$} & \multirow{2}{*}{$\begin{array}{l}\text { Fim } \\
\text { pega } \\
\text { (h) }\end{array}$} & \multirow{2}{*}{\multicolumn{2}{|c|}{$\begin{array}{c}\text { Água de } \\
\text { cons. de } \\
\text { pasta (\%) }\end{array}$}} & \multirow{2}{*}{\multicolumn{2}{|c|}{$\begin{array}{c}\text { Superf. } \\
\text { Blaine } \\
\left(\mathrm{m}^{2} / \mathrm{kg}\right)\end{array}$}} & \multicolumn{2}{|c|}{ Finura (\%) } & \multicolumn{3}{|c|}{$\begin{array}{c}\text { Resistência à } \\
\text { compressão (MPa) }\end{array}$} \\
\hline & & & & & & & \#200 & \#325 & $\begin{array}{c}3 \\
\text { dias }\end{array}$ & $\begin{array}{c}7 \\
\text { dias }\end{array}$ & $\begin{array}{l}28 \\
\text { dias }\end{array}$ \\
\hline 3,11 & $03: 29$ & $04: 11$ & 26,6 & & & & 2,15 & 11,35 & 28,0 & 34,2 & 41,1 \\
\hline
\end{tabular}

Fonte: Relatório de ensaio Itambé - mar/2015

O agregado miúdo utilizado no estudo é uma areia industrial, obtida por britagem de rocha granítica. Como agregado graúdo, são utilizadas brita 0 e brita 1, de origem calcária dolomítica e basáltica, respectivamente. O cimento Portland utilizado na pesquisa é o CP II-F-32, comumente usado na produção de concreto convencional dosado em central. Para efetuar a alteração da relação agregados secos/cimento, mantendo fixo o fator a/c e abatimento, foram utilizados os aditivos polifuncional MasterPolyheed 38 (plastificante) da BASF e o Power Flow 1180 (superplastificante) da MC-Bauchemie.

Os concretos foram dosados experimentalmente e caracterizados em laboratório. Foram elaboradas duas famílias de curvas de dosagem para cada classe. A primeira para os agregados em sua condição natural, adotando a areia e a brita 1 como agregados. Esta família de curvas foi utilizada como referência. A proporção entre areia e brita 1 foi estabelecida segundo teor ótimo de argamassa, conforme proposto pelo método do IPT/EPUSP [24]. A segunda família foi produzida com os mesmos agregados, adicionando-se ainda a brita 0 . Adotou-se o método de dosagem proposto por Tutikian e Dal Molin [25] para concretos autoadensáveis, sendo este baseado no método de dosagem IPT/EPUSP [24]. O empacotamento dos três agregados foi feito experimentalmente, seguindo os procedimentos descrito por O'Reilly [26].

A partir dos diagramas de dosagem confeccionados para as duas famílias de concreto, foram definidos os traços para as duas classes de resistência à compressão a serem estudadas (C25 e C40) resultando em 4 traços de concreto a serem estudados ( 1 traço de referência, 1 traço em que o esqueleto granular foi modificado pelo empacotamento de partículas com a mesma resistência do traço referência e 2 traços em que a partir do esqueleto granular modificado pelo empacotamento de partículas variou-se o valor da relação agregado seco/cimento), apresentados na Tabela 02. 
LENZ, L. A.;ET AL.. AVALAÇÃO DA INFLUÊNCIA DO EMPACOTAMENTO DOS AGREGADOS NA DURABILIDADE DE CONCRETOS ATRAVÉS DE ENSAIOS NÃO DESTRUTIVOS: RESISTIVIDADE ELÉTRICA E PROPAGAÇÃO DE ONDAS DE ULTRASSOM. $2^{\circ}$ Simpósio Paranaense de Patologia das Construções (20 SPPC), artigo 2SPPC1016, pp. 185-198, 2017. DOI:

Tabela 02: Traços para os concretos produzidos

\begin{tabular}{cccccc}
\hline Classe & $\begin{array}{c}\text { Estr. } \\
\text { granular }\end{array}$ & $\mathbf{m}$ & Traço & $\mathbf{a} / \mathbf{c}$ & $\begin{array}{c}\mathbf{C}_{\text {cim }} \\
\left(\mathbf{k g} / \mathbf{m}^{3}\right)\end{array}$ \\
\hline \multirow{2}{*}{ C25 } & Referência & 4,36 & $1: 1,84: 2,52$ & 0,53 & 409,54 \\
\cline { 2 - 6 } & Modificada & 5,08 & $1: 2,54: 1,52: 1,02$ & 0,58 & 360,28 \\
\hline \multirow{2}{*}{ C40 } & Referência & 2,73 & $1: 0,98: 1,75$ & 0,41 & 550,09 \\
\cline { 2 - 6 } & Modificada & 3,08 & $1: 1,54: 0,92: 0,62$ & 0,41 & 535,27 \\
\hline
\end{tabular}

Na sequência, como forma de avaliar a influência da quantidade de agregados em relação à pasta na resistividade elétrica e na propagação das ondas ultrassônicas, variou-se a relação agregados secos/cimento, partindo-se do teor definido para os traços $\mathrm{C} 25$ e C40 com a estrutura granular modificada. Assim, utilizaram-se valores iguais a 5,08; 6,08 e 7,08 para os concretos de classe C25 e 3,08; 4,08 e 5,08 para os concretos de classe $\mathrm{C} 40$. Os valores iniciais da relação agregados secos/cimentopara as duas classes de concreto foram definidas a partir da dosagem e produção dos concretos com estrutura granular modificada.

A Tabela 03 apresenta os traços para os concretos produzidos, o consumo de cimento e os teores de aditivo plastificante (P) e superplastificante (SP).

Tabela 03: Variação do teor de agregado $(\mathrm{m})$ nos concretos: traços produzidos

\begin{tabular}{|c|c|c|c|c|c|c|}
\hline Classe & m & Traço & $a / c$ & $\begin{array}{c}\mathbf{P} \\
(\%)\end{array}$ & $\begin{array}{l}\text { SP } \\
(\%)\end{array}$ & $\begin{array}{c}\mathrm{C}_{\text {cim }} \\
\left(\mathrm{kg} / \mathrm{m}^{3}\right)\end{array}$ \\
\hline \multirow{3}{*}{ C25 } & 5,08 & $1: 2,54: 1,52: 1,02$ & \multirow{3}{*}{0,58} & - & - & 360,28 \\
\hline & 6,08 & $1: 3,04: 1,82: 1,22$ & & \multirow{2}{*}{0,8} & 1,35 & 314,10 \\
\hline & 7,08 & $1: 3,54: 2,12: 1,42$ & & & 1,90 & 277,83 \\
\hline \multirow{3}{*}{ C40 } & 3,08 & $1: 1,54: 0,92: 0,62$ & \multirow{3}{*}{0,41} & - & - & 535,27 \\
\hline & 4,08 & $1: 2,04: 1,22: 0,82$ & & \multirow{2}{*}{0,8} & 1,52 & 439,53 \\
\hline & 5,08 & $1: 2,54: 1,52: 1,02$ & & & 2,35 & 371,80 \\
\hline
\end{tabular}

Os traços foram dosados mantendo-se fixa a relação a/c, igual a 0,58 e 0,41 para os concretos de classe C25 e C40, respectivamente. A quantidade de aditivo superplastificante foi sendo variada para atingir o abatimento de $(100 \pm 10) \mathrm{mm}$, sem ultrapassar a dosagem recomendada pelo fabricante de 0,2 a $5 \%$ sobre o peso do cimento. Observou-se que a medida que se aumentava a porcentagem de aditivo superplastificante, alterava-se a reologia do concreto, tornando-o mais coeso sem, entretanto, levar a segregação do conjunto.

A produção dos concretos apresentados na Tabela 02 e 03 foram executadas em laboratório, em betoneira com capacidade de 250 litros, seguindo a ordem de mistura de materiais recomendada por Helene [24]. Esta consiste em misturar inicialmente $80 \%$ da água com o agregado graúdo, seguido pelo agregado miúdo, cimento e o restante da água. A mistura ocorreu em sequência com intervalos de 2 minutos entre a adição de cada material, garantindo a completa homogeneização. Por fim, foram moldados os corpos de prova necessários para os ensaios mecânicos e de durabilidade, resultando em 40 corpos de prova cilíndricos no total, todos com dimensões de $10 \times 20 \mathrm{~cm}$, moldados em forma metálica e com aplicação de desmoldante. $\mathrm{O}$ adensamento do concreto foi realizado manualmente, conforme recomendações da NBR 5738 [27]. 
LENZ, L. A.;ET AL.. AVALAÇÃO DA INFLUÊNCIA DO EMPACOTAMENTO DOS AGREGADOS NA DURABILIDADE DE CONCRETOS ATRAVÉS DE ENSAIOS NÃO DESTRUTIVOS: RESISTIVIDADE ELÉTRICA E PROPAGAÇÃO DE ONDAS DE ULTRASSOM. $2^{\circ}$ Simpósio Paranaense de Patologia das Construções (2० SPPC), artigo 2SPPC1016, pp. 185-198, 2017. DOI: 10.4322/2SPPC.2017.016

A resistividade elétrica dos concretos estudados foi mensurada com auxílio do equipamento RESIPOD (PROCEQ AS) pelo método de Wenner [18]. Neste ensaio, foram utilizados os mesmos corpos de prova utilizados no ensaio de ultrassom, para determinação do módulo de elasticidade dinâmico. Foram realizadas leituras na idade de 28 dias com os corpos de prova saturados em água.

Para a determinação da velocidade de propagação de ondas de ultrassom, utilizouse o equipamento Pundit PL-200, da Proceq. O procedimento foi adotado conforme as recomendações da NBR 8802 [21] e empregou-se a transmissão direta entre os transdutores, com frequência de onda de $54 \mathrm{kHz}$ e propagação de onda a cada 9,3 $\mu \mathrm{s}$.

Aplicou-se a técnica de análise estatística de variância ANOVA para os resultados das amostras de concreto classe C25 e C40, separadamente e em conjunto. A metodologia do teste consiste na aplicação do Teste de Turkey [28]. Para confirmar as resistências à compressão dos concretos produzidos, seguiu-se os procedimentos descritos na NBR 5739 [29]. O ensaio de compressão axial foi realizado com a utilização de neoprene para regularização das bases dos corpos de prova e aplicação uniforme da carga. O ensaio foi realizado em prensa EMIC com capacidade total de carga de 200 tf.

\section{Resultados e Discussão}

\subsection{Resistência à compressão}

Os resultados de massa específica e resistência à compressão dos concretos estudados estão indicados na Tabela 04. Ressalta-se que os resultados apresentados são uma média de cinco corpos de prova.

Tabela 04: Resultados de resistência à compressão dos concretos estudados

\begin{tabular}{|c|c|c|c|c|c|c|}
\hline$n^{\circ}$ & Classe & $\begin{array}{c}\text { Estrutura } \\
\text { granular }\end{array}$ & $\mathbf{m}$ & $\begin{array}{c}\text { Massa } \\
\text { específica } \\
\left(\mathrm{kg} / \mathrm{m}^{3}\right)\end{array}$ & $\begin{array}{c}F_{c d} \\
(\mathrm{MPa})\end{array}$ & $\begin{array}{c}\text { Desv. Pad. } \\
\text { (Mpa) }\end{array}$ \\
\hline 1 & \multirow{4}{*}{ C25 } & Referência (Ref.) & 4,36 & 2380 & 34,7 & 1,7 \\
\hline 2 & & \multirow{3}{*}{$\begin{array}{l}\text { Modificada } \\
\text { (Emp.) }\end{array}$} & 5,08 & 2406 & 34,5 & 1,2 \\
\hline 3 & & & 6,08 & 2408 & 41,2 & 0,7 \\
\hline 4 & & & 7,08 & 2410 & 28,7 & 1,4 \\
\hline 5 & \multirow{4}{*}{ C40 } & Referência (Ref.) & 2,73 & 2370 & 49,4 & 1,4 \\
\hline 6 & & \multirow{3}{*}{$\begin{array}{l}\text { Modificada } \\
\text { (Emp.) }\end{array}$} & 3,08 & 2400 & 49,2 & 1,6 \\
\hline 7 & & & 4,08 & 2450 & 49,0 & 1,7 \\
\hline 8 & & & 5,98 & 2445 & 41,1 & 0,7 \\
\hline
\end{tabular}

Com base na Tabela 04 e na análise de variância, observa-se que não existem diferenças significativas entre as resistências à compressão dos concretos pertencentes à mesma classe, C25 (concretos 1 e 2) ou C40 (concretos 5 e 6), para a idade de 28 dias, ao efetuar somente a modificação do esqueleto granular pelo empacotamento de partículas. Isso já era esperado, pois a dosagem realizada buscou produzir concretos com a mesma resistência à compressão, tornando possível avaliar os efeitos da modificação do esqueleto granular na resistividade elétrica e módulo de 
elasticidade dinâmico no concreto. Já à medida que se aumenta o empacotamento dos agregados (o valor da relação agregado seco/cimento) a partir dos concretos 2 (C25) e 6 (C40), mesmo com a redução de cimento, a resistência aumentou até certo limite e, após este, ocorreu a queda. Isso deve-se ao fato que até um certo limite o travamento dos agregados consegue aumentar a resistência mecânica à compressão, pois as partículas do agregado se aproximam mais umas das outras. A partir deste limite, porém, falta pasta de cimento para preencher os espaços entre as partículas, já que a pasta pode não conseguir penetrar em todos os espaços entre partículas, devido ao teor elevado de grãos.

\subsection{Resistividade elétrica}

Os resultados do ensaio de resistividade elétrica dos concretos estudados na idade de 28 dias seguem apresentados na Fig. 01. Os resultados apresentados são uma média de 5 corpos de prova.

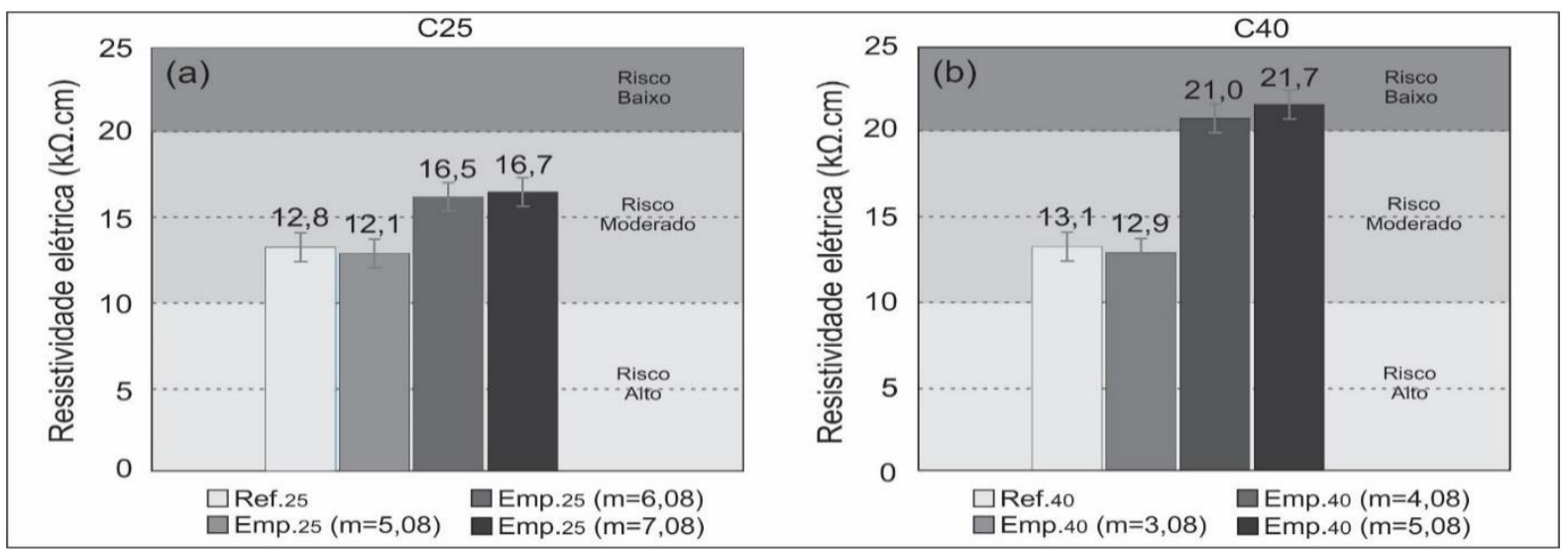

Figura 01: Resistividade elétrica dos concretos estudados aos 28 dias: (a) classe C25 e (b) classe C40

Ao comparar o concreto de referência Ref.25 e Ref.40 com o de estrutura granular modificada pelo empacotamento Emp.25 ( $m=5,08)$ e Emp.40 ( $m=3,08)$ com mesma resistência mecânica é possível constatar a partir da Fig.01 que ao modificar a estrutura granular dos concretos ocorre a redução do valor da resistividade elétrica. A redução dos concretos C25 é de 5,5\% e dos C40 é de 1,52\%. Ao aplica-se o método ANOVA de análise de variância foi possível constatar que para ambos os casos os resultados são considerados estatisticamente equivalentes.

Observa-se ainda pela Fig. 01 que todos os concretos de classe C25 apresentam resultados de resistividade elétrica entre 10 a $20 \mathrm{k} \Omega . \mathrm{cm}$, apresentando risco moderado de corrosão de armadura, segundo classificação dada por Whiting e Nagi [14] para 28 dias. Já para os concretos classe C40, dois deles alcançaram resistividade elétrica acima de $20 \mathrm{k} \Omega . \mathrm{cm}$, o concreto Emp.40 (m=4,08) e Emp.40 $(m=5,08)$, que corresponde a um risco baixo de corrosão.

Nota-se para as duas classes de concretos que, ao aumentar a relação agregados secos/cimento, há um significativo aumento na resistividade elétrica. Entretanto, do Emp.25 $(m=6,08)$ para o Emp.25 $(7,08)$ e do Emp.40 $(4,08)$ para Emp.40 $(5,08)$, percebe-se que os resultados são estatisticamente iguais, com diferenças de médias 
LENZ, L. A.;ET AL.. AVALAÇÃO DA INFLUÊNCIA DO EMPACOTAMENTO DOS AGREGADOS NA DURABILIDADE DE CONCRETOS ATRAVÉS DE ENSAIOS NÃO DESTRUTIVOS: RESISTIVIDADE ELÉTRICA E PROPAGAÇÃO DE ONDAS DE ULTRASSOM. $2^{\circ}$ Simpósio Paranaense de Patologia das Construções (20 SPPC), artigo 2SPPC1016, pp. 185-198, 2017. DOI: 10.4322/2SPPC.2017.016

menores que $3,5 \%$, demonstrando assim que a partir de um ponto, o aumento no teor de agregados causa pouco incremento na resistividade elétrica do concreto.

\subsection{Módulo de Elasticidade Dinâmico através do ensaio de Ultrassom}

O ensaio de velocidade de propagação de ondas ultrassônicas foi realizado aos 28 dias, e os resultados da média das velocidades obtidas encontram-se no gráfico da Fig. 02. Todas as leituras foram feitas com os corpos de prova na condição saturada superfície seca. Os resultados apresentados são uma média de 5 corpos de prova.

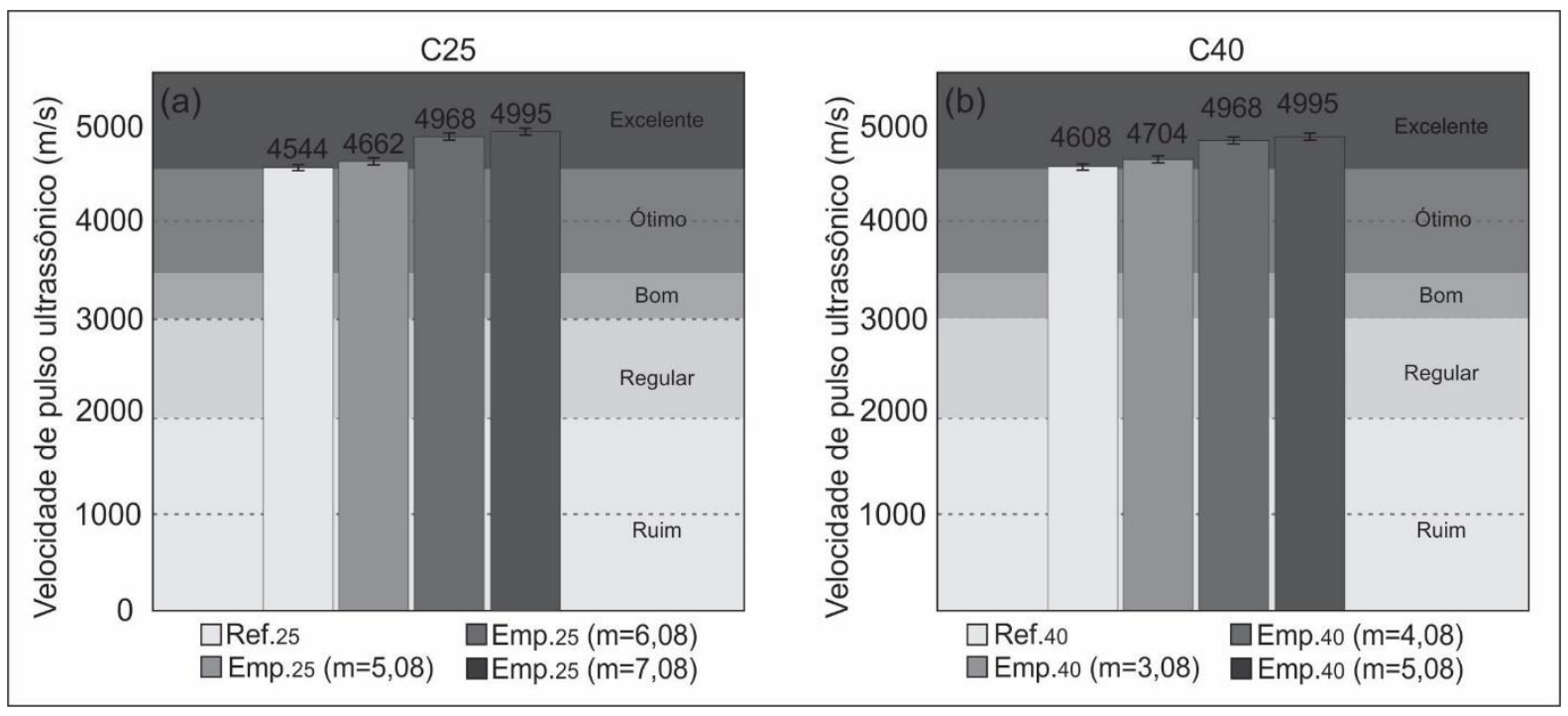

Figura 02: Velocidade de pulso ultrassônico dos concretos estudados aos 28 dias:

(a) classe C25 e (b) classe C40

É possível constatar pela Fig.02 que as velocidades de pulso ultrassônico foram superiores a $4500 \mathrm{~m} / \mathrm{s}$ para todos os concretos, sendo classificados assim como de qualidade excelente, conforme relação estabelecida por Cánovas [30]. O concreto de referência apresentou velocidades inferiores quando comparada ao concreto com estrutura granular modificada pelo empacotamento de partículase, aplicando 0 método ANOVA de análise de variância, foi possível constatar que todos os resultados apresentam diferenças significativas uns dos outros. Isso ocorreu porque, em geral, os agregados graúdos e miúdos têm módulo de elasticidade maior, e, por consequência, maior velocidade de propagação da onda de ultrassom do que a pasta de cimento [31]. Desta forma, como os concretos com estrutura granular modificada possuem uma maior quantidade de agregado, apresentam maior velocidade de propagação do pulso ultrassônico.

Ao comparar os concretos com estrutura granular modificada pelo empacotamento uns com os outros, observou-se que as velocidades de pulso ultrassônico foram aumentando conforme a relação agregados secos/cimento foi incrementada, ou seja, conforme se aumentou o valor de $\mathrm{m}$. Hernández et al. [32] comentam que quanto menor o volume de vazios no interior do concreto, maior é a velocidade de propagação do pulso ultrassônico, pois a velocidade de propagação no ar é menor do que nos sólidos. Logo, o aumento do teor de agregados colaborou para reduzir o índice de vazios, o que reforça os resultados obtidos pelo ensaio de resistividade elétrica. 
Com os resultados de velocidade de pulso ultrassônico obtidos foi possível calcular o módulo de elasticidade dinâmico dos concretos aos 28 dias, conforme mostra a Fig. 03. Para o cálculo considerou-se um coeficiente de Poisson igual a 0,2 e a massa específica no estado endurecido dos corpos de prova.

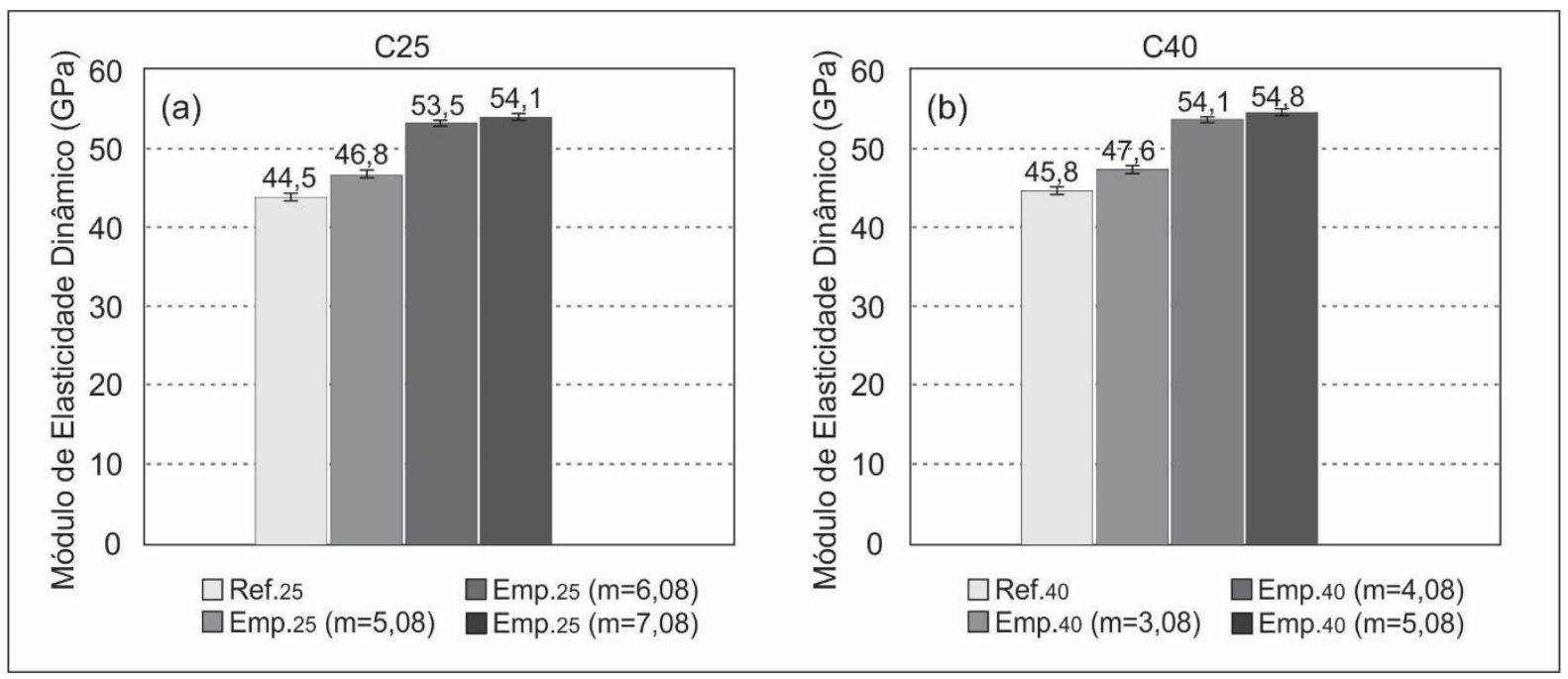

Figura 03: Módulo de elasticidade dinâmico dos concretos estudados aos 28 dias:

(a) classe C25 e (b) classe C40

Ao comparar os concretos Ref.25 com Emp.25 ( $m=5,08)$ e Ref.40 com Emp.40 $(3,08)$ observa-se pela Fig. 03 que o concreto com estrutura granular modificada atingiu os maiores valores de módulo de elasticidade dinâmico, alcançando 46,8 GPa e 47,6 GPa aos 28 dias para os concretos classe C25 e C40, respectivamente, resultado 5\% e $4 \%$ superior ao concreto de referência. Ao aplicar o teste de variância, constatouse que ambos os concretos apresentam diferenças significativas nos resultados de módulo de elasticidade dinâmico, o que demonstra que ao efetuar a modificação do esqueleto granular houve um incremento dos resultados. Logo, pode-se concluir que esses concretos apresentaram maior rigidez do que o concreto de referência, ou seja, menor é a capacidade de deformação. Esse resultado pode ser explicado pelo fato de que os concretos com estrutura granular modificada apresentam um empacotamento mais eficiente, com a inserção da brita 0 e a mudança na proporção entre os agregados.

Ao comparar os concretos com estrutura granular modificada pelo empacotamento uns com os outros, observa-se a partir da Fig. 03 que à medida que se aumenta a relação agregados secos/cimento, aumenta-se o módulo de elasticidade dinâmico. Para o concreto classe C25, verificou-se incremento de até $16 \%$ no módulo de elasticidade. Para o concreto classe $\mathrm{C} 40$, os valores aumentaram em até $15 \%$. Ao aplicar o teste de variância constatou-se que os concretos Emp.25 ( $m=6,08)$ e Emp.25 $(m=7,08)$ assim como Emp.40 ( $m=4,08)$ e Emp.40 $(m=5,08)$ podem ser considerados estatisticamente equivalentes. Dessa forma, pode-se concluir que esses concretos apresentam mais rigidez do que o Emp.25 ( $m=5,08)$ e Emp.40 ( $m=3,08)$. Ou seja, menor é a capacidade de deformação. Esse resultado pode ser justificado pelo fato de que esses concretos apresentam menos vazios e mais agregados que os de Emp.25 $(m=5,08)$ e Emp.40 $(m=3,08)$, com diminuição da quantidade de pasta. 


\section{Conclusões}

Após a realização dos experimentos propostos e análise dos resultados obtidos, as seguintes conclusões podem ser delineadas:

- Os ensaios de resistividade elétrica e velocidade de pulso ultrassônico dos concretos mostraram que, conforme se aumenta a relação agregados secos/cimento $(\mathrm{m})$, a porosidade do material diminui. $\mathrm{O}$ bom desempenho desses concretos pode ser atribuído à densificação que o incremento do esqueleto granular proporciona, já que a rocha que forma os agregados é, em geral, menos porosa que a matriz de cimento dos concretos convencionais.

- Os resultados das velocidades de pulso ultrassônico foram superiores a $4500 \mathrm{~m} / \mathrm{s}$ para todos os concretos, indicando que os concretos são de qualidade excelente. Os concretosRef.25 e Ref.40 apresentaram velocidades inferiores quando comparada ao concreto com estrutura granular modificada pelo empacotamento de partículas. Os resultados do módulo de elasticidade dinâmico do concreto com a estrutura granular modificada para ambas as classes foram superiores aos de referência, sendo as diferenças estatisticamente significativas. Logo, pode-se concluir que esses concretos apresentaram maior rigidez do que o concreto de referência, ou seja, menor é a capacidade de deformação. Esse resultado pode ser explicado pelo fato de que os concretos com estrutura granular modificada apresentam um empacotamento mais eficiente, com a inserção da brita 0 e a mudança na proporção entre os agregados.

\section{Agradecimentos}

Os autores gostariam de agradecer à empresa Concrebras, que forneceu o material necessário à realização dos experimentos apresentados neste trabalho.

\section{Referências}

[1] Neville, A.; Brooks, J. J. Tecnologia do concreto. 2 ed. Porto Alegre: Bookman, 2013.

[2] NBR 6118 (2014) Projetos de Estrutura de Concretos - Procedimentos. Associação Brasileira de Normas Técnicas - ABNT, São Paulo, ABNT, 2014.

[3] Mehta, P. K.; Monteiro, P. Concreto, estrutura, propriedades e materiais. São Paulo, IBRACON, 2008.

[4] Wong, V.; Chan, K.W.; Kwan, A. K. H. Applying theories of particle packing and rheology to concrete for sustainable development. Org. Technol. Manag. Constr. n. 5, p. 844-851. 2013.

[5] De Larrard, F. Concrete mixture proportioning: a scientific approach. Modern Concrete Technology Series, n. 9. E\&FN SPON, London, 1999.

[6] Vanderlei, R. D. Análise experimental do concreto de pós reativos: dosagem e propriedades mecânicas. 2004.168 f. Tese Doutorado em Engenharia de 
LENZ, L. A.;ET AL.. AVALAÇÃO DA INFLUÊNCIA DO EMPACOTAMENTO DOS AGREGADOS NA DURABILIDADE DE CONCRETOS ATRAVÉS DE ENSAIOS NÃO DESTRUTIVOS: RESISTIVIDADE ELÉTRICA E PROPAGAÇÃO DE ONDAS DE ULTRASSOM. $2^{\circ}$ Simpósio Paranaense de Patologia das Construções (20 SPPC), artigo 2SPPC1016, pp. 185-198, 2017. DOI: 10.4322/2SPPC.2017.016

Estruturas. Escola de Engenharia de S. Carlos, Universidade de S. Paulo, S. Carlos, SP, 2004.

[7] Funk, J.E., Dinger, D.R. Particle packing, part II: Review of packing of polydisperse particle systems. In: Interceram, p.95-97, 1992.

[8] Kwan, A. K. H.; Mora, C. F. Effects of various shape parameters on packing of aggregate particles. Magazine of Concrete Research. 2001.

[9] Fennis, S.A.A.M.; Walraven, J.C.; DEN UIJL, J.A. Compaction-interaction packing model: regarding the effect of fillers in concrete mixture design, Materials and Structures, v. 46, p. 463-478, 2013a.

[10] Filho, P. F.; Pereira, J. S. Tendências Atuais de Inspeção de Equipamentos Estáticos, São Paulo, 2005.

[11] Helene, P. R. L. Contribuição ao estudo de corrosão de armaduras de concreto armado. Tese de Livre Docência, EPUSP, São Paulo, 1993.

[12] Polder, R. B. Test method for on site measurement of resistivity of concrete - a RILEM TC-154 technical recomendation. Construction and Building Materials. v. 15, p. 125-131, 2001.

[13] Cascudo, O. O Controle da Corrosão de Armaduras em Concreto, Inspeção e técnicas eletroquímicas. Editora UFG, Goiânia, 1997.

[14] Whiting, D.A.; Nagi, M. A. E. Electrical Resistivity of Concrete - A Literature Review. Skokie/USA, Portland Cement Association, 57 p. 2003.

[15] Hoppe, T. F. Resistividade elétrica de concretos contendo diferentes teores de cinza de casca de arroz. Dissertação de Mestrado - Universidade Federal de Santa Maria - Centro de Tecnologia - Programa de Pós Graduação em Engenharia Civil, Santa Maria, Rio Grande do Sul, 2005.

[16] Chen, C.; Chang, J.; Yeih, W.The effects of specimen parameters on the resistivity of concrete. Construction and Building Materials. n. 71, p. 35-43. 2014.

[17] Santos, L. Avaliação da resistividade elétrica do concreto como parâmetro para a previsão da iniciação da corrosão induzida por cloretos em estruturas de concreto. Dissertação (Mestrado em estruturas e construção civil), Universidade de Brasília. Brasília, DF, 161p. 2006.

[18] Millard, S.G. Reinforced Concrete Resistivity Measurement Techniques. Institution of Civil Engineers, Parte 2: Research and Theory. Proceeding. v 91, n. 2, Mar, 1991, p.71-88.

[19] Evangelista, A. C. J. Avaliação da resistência do concreto usando diferentes ensaios não destrutivos. Tese (Doutorado em Engenharia Civil) - Universidade Federal do Rio de Janeiro. COPPE, Rio de Janeiro, 2002. 219p.

[20] Silva, A. P.; Segadães, A. M.; Deezas, T. C. Relações entre distribuição granulométrica, morfologia e empacotamento de partículas num sistema real: alta alumina. In Congresso Brasileiro de Cerâmica, 47ํㅗ , João Pessoa-PB, 2003. Anais do $47^{\circ}$ Congresso Brasileiro de Cerâmica, 2003. 
LENZ, L. A.;ET AL.. AVALAÇÃO DA INFLUÊNCIA DO EMPACOTAMENTO DOS AGREGADOS NA DURABILIDADE DE CONCRETOS ATRAVÉS DE ENSAIOS NÃO DESTRUTIVOS: RESISTIVIDADE ELÉTRICA E PROPAGAÇÃO DE ONDAS DE ULTRASSOM. $2^{\circ}$ Simpósio Paranaense de Patologia das Construções (2० SPPC), artigo 2SPPC1016, pp. 185-198, 2017. DOI:

[21] NBR 8802: Concreto endurecido - Determinação da velocidade de propagação de onda ultrassônica. Associação Brasileira de Normas Técnicas - ABNT, Rio de Janeiro, Brasil. 1997.

[22] ASTM C 597 (2009) Standard Test Method for Pulse Velocity Through Concrete. American Society for testing and material, West Conshohocken, U.S.

[23] NBR 9833: Concreto fresco - Determinação da massa específica, do rendimento e do teor de ar pelo método gravimétrico. Rio de Janeiro. 2008. 3p.

[24] Helene, P.; Terzian, P. Manual de dosagem e controle do concreto. São Paulo: PINI, 1993. 348p.

[25] TUTIKIAN, B. F.; DAL MOLIN, D. C. Concreto auto-adensável. $1^{\circ}$ ed. São Paulo: PINI, 2008.

[26] O'Reilly, V.D. Método de dosagem de concreto de elevado desempenho. Pini, São Paulo, 1992.

[27] NBR 5738: Concreto - moldagem e cura de corpos-de-prova cilíndricos ou prismáticos de concreto. Rio de Janeiro, 2015.

[28] Vieira, S. Análise de variância: ANOVA. Atlas, São Paulo, 2006.

[29] NBR 5739: Concreto - Ensaio de compressão de corpos-de-prova cilíndricos. Rio de Janeiro, 1994.

[30] Cananovas, M. F. Patologia e Terapia do Concreto. São Paulo. Pini, 1998.

[31] Chung, H. W.; Law, K. S. Diagnosing in situ concrete by ultrasonic pulse technique, Concrete International, n. 5, p.42-49, 1985.

[32] Hernández, M. G.; Izquierdo, M. A. G.; Ibãnés, A.; Anaya, J. J.; Ullate, L. G. Porosity estimation of concrete by ultrasonic NDE. Ultrasonics. v. 38, p. 531-533. 2000. 